\begin{tabular}{llll} 
Abstract E-095 Table 1 & & \\
\hline Year & Room & $\begin{array}{l}\text { Mean DTA minutes } \\
\text { (number of patients) }\end{array}$ & $\begin{array}{l}\text { Mean ITA } \\
\text { minutes }\end{array}$ \\
\hline 2017 & Biplane Suite & $116(88)$ & 86 \\
2018 first half & Biplane Suite & $96(72)$ & 77 \\
2018 second half & Biplane Suite & $94(27)$ & 66 \\
2019 first half & Biplane Suite & $81(21)$ & 64 \\
2018 second half & Hybrid Suite & $108(18)$ & 78 \\
2019 first half & Hybrid Suite & $100(33)$ & 66 \\
\hline
\end{tabular}

Methods Data are from a single center's neurointerventional database from January 2017 to July 2019, excluding in-house stroke patients and patients who had imaging at an outside hospital. Workflow metrics acquired were: door to arterial access (DTA) and CT first image acquisition to arterial access (ITA). Data are described by calendar year and half-years and subdivided by two different angiography suites: the usual neuroangiography biplane suite (BS) located distant to the emergency room (ER) and the hybrid suite (HS) immediately adjacent to the ER. The details of the quality improvement initiative were obtained from internal organizational documents as were the details of the training required for the opening of the hybrid room and the subsequently encountered problems.

Results After the launch of a modest stroke quality improvement initiative in January 2018 until July $2019(n=208)$, both average DTA and ITA decreased every 6 months in the BS, from 116 to 81 minutes and 86 to 64 minutes, respectively. Despite extensive training and preparation, the opening of the HS in the second half of 2018 showed worse DTA and ITA not only for the first 6 months (18 patients) but also for the first half of 2019 (33 patients) compared to the BS although improvement was demonstrated over the first year of operation. Several setbacks and challenges were associated with the HS most notably with the monoplane robotic C-arm and monitor.

Conclusion A workflow quality improvement initiative had a durable effect on reducing EVT treatment times overall and particularly in the existing, geographically distant biplane suite, however, the new lower times were not reproducible within the complicated infrastructure of a new modern hybrid suite next to the ER.

Disclosures K. Blackham: None. G. Karwacki: None. D. Zumofen: None. T. Schubert: None. M. Psychogios: None.

\section{E-096 EMS PROVIDER PREFERENCE COMPARISON BETWEEN THE PRE-HOSPITAL LVO DETECTION DURING INITIAL CARE TRANSPORT (PREDICT) SCALE AND THE RAPID ARTERIAL OCCLUSION EVALUATION (RACE) SCALE: A SUBANALYSIS OF THE PREDICT STUDY}

\footnotetext{
${ }^{1} \mathrm{E}$ Fortuny ${ }^{*},{ }^{1} \mathrm{~A}$ Cruz, ${ }^{1} \mathrm{~B}$ Ugiliweneza, ${ }^{1} \mathrm{D}$ Wang, ${ }^{2} \mathrm{~A}$ White, ${ }^{1} \mathrm{~N}$ Khattar, ${ }^{1} \mathrm{~B}$ Gallinore, ${ }^{1} \mathrm{D}$ Ding, ${ }^{3} \mathrm{~S}$ Wolfe, ${ }^{4} \mathrm{D}$ Heck, ${ }^{1} \mathrm{R}$ James. ${ }^{1}$ Neurosurgery, University of Louisville, Louisville, KY; ${ }^{2}$ Radiology, University of Louisville, Louisville, KY; ${ }^{3}$ Neurosurgery, Wake Forest Baptist Health, Lexington, NC; ${ }^{4}$ Radiology, Forsythe Medical Center, Winston-Salem, NC
}

\subsection{6/neurintsurg-2020-SNIS. 129}

Intro Accurate identification of large vessel occlusion (LVO) strokes in the field may save lives through reduced transport times. Emergency Medical Services (EMS) training is critical to the successful implementation of prehospital screening scales. The prospectively validated 6-item Rapid Arterial oCclusion Evaluation (RACE) scale is a common EMS LVO stroke scale. As part of our prospective, pre-hospital EMS Validation comparison study of the PRe-hospital ELVO Detection during Initial Care Transport (PREDICT) scale, we hypothesized that EMS providers without prior exposure to RACE would prefer the shorter, simpler PREDICT-4 scale and due to the cognitive bias known as the 'mere exposure effect,' EMS providers with previous experience with RACE will prefer it over the new PREDICT scale.

Methods A total of 86 EMS providers were asked their preferred scale after performing both scales on a subject suspected of having a stroke. EMS providers were trained on a secure, online application to perform the PREDICT-4 scale and record both scales and complete a one-question survey (figure 1). A chi-square test and one-sample test of proportion was conducted to determine the preferred scale.

Results 86 EMS providers enrolled a total of 250 subjects. 38 providers enrolled only one subject and 48 enrolled 2 or more. When comparing scale preference of all studied EMS providers (both with and without previous exposure to the RACE scale prior to this study), $37.2 \%$ preferred the PREDICT 4-item scale and $62.8 \%$ preferred the RACE scale, $\mathrm{p}=0.018,95 \%$ CI $[27.7,47.8$ and 52.2, 72.2, respectively]. The planned subgroup analysis of EMS providers who did not have prior mere-exposure to the RACE scale $(n=49)$, we found that $34.7 \%$ preferred the PREDICT 4-item scale [95\% CI 22.9, 48.6\%] and $65.3 \%$ preferred the RACE scale $\mathrm{p}=0.03,95 \%$ CI $[51.3,77.0]$. In the subgroup analysis of the 48 providers who enrolled $\geq 2$, on their first screening 20 (41.6\%, p $=0.248,95 \%$ CI $[28.8,55.7])$ preferred PREDICT-4 and $28(58.3 \%)$ RACE. On their last screening, only 18 (37.5\%, p=0.08, 95\% CI [25.2, 51.6]) providers preferred PREDICT-4. 
Conclusion The shorter and simpler PREDICT scale was found to be less preferable with EMS provider all-comers, which may be partially due to the mere-exposure cognitive bias effect though this difference persisted in providers who had no prior exposure to either scale. In providers who enrolled multiple patients, there was a statistically insignificant trend towards preference for RACE between their first and last screening.

Disclosures E. Fortuny: None. A. Cruz: None. B. Ugiliweneza: None. D. Wang: None. A. White: None. N. Khattar: None. B. Gallinore: None. D. Ding: None. S. Wolfe: None. D. Heck: None. R. James: 1; C; Medtronic.

\section{E-097 TECHNICAL OUTCOMES OF MECHANICAL THROMBECTOMY USING THE SOFIA PLUS ASPIRATION CATHETER}

A Witek*, T Patterson, L Sheikhi, M Bain, M Hussain, G Toth. Cerebrovascular Center, Cleveland Clinic, Cleveland, $\mathrm{OH}$

\subsection{6/neurintsurg-2020-SNIS. 130}

Introduction The Sofia Plus aspiration catheter (Microvention; Tustin, CA) is a recently introduced 0.070-inch inner diameter catheter designed to provide distal intracranial access and engage thrombus to facilitate thrombectomy for acute ischemic stroke.

Methods This single-center retrospective study assessed technical outcomes of mechanical thrombectomy using the Sofia Plus aspiration catheter. Utilizing a prospective database of all acute stroke interventions at our institution, cases utilizing SOFIA Plus on the first pass were identified. Patient characteristics, procedural details, and radiographic outcomes were analyzed.

Results Sofia Plus was utilized as a first pass aspiration catheter in 138 cases. Patients presented with a median NIHSS of 17 (IQR 10-22), ASPECT of 10 (IQR 8-10), and had target occlusions involving the ICA $(n=30)$, M1 $(n=73)$, M2 $(n=22)$, or basilar artery $(n=13)$. The aspiration catheter was used for first-pass direct aspiration alone in 86 patients $(62 \%)$, and in conjunction with a stent retriever in 52 patients $(38 \%)$. A recanalization grade of TICI $2 \mathrm{~b}$ or better was achieved in 118 patients (86\%), which was accomplished with a single pass in 88 (64\%). Median time from groin puncture to recanalization was 44 minutes (IQR 30-68). Comparison of direct aspiration alone versus with stent retrievers demonstrated no difference in recanalization grade $(\mathrm{p}=0.64)$ or rates of first-pass recanalization $(p=0.28)$, but direct aspiration was associated with faster recanalization (41 vs 53 minutes, $\mathrm{p}=0.02$ ). There were two cases of vessel perforation (both involving stent retrievers), and two additional patients had symptomatic ICH on follow-up CT.

Conclusions Our experience demonstrates that the SOFIA Plus aspiration can facilitate safe and effective revascularization for acute ischemic stroke, either using a direct aspiration technique or in conjunction with a stent-retriever. However, reperfusion was achieved faster with direct aspiration alone.

Disclosures A. Witek: None. T. Patterson: None. L. Sheikhi: None. M. Bain: 2; C; Medtronic PLC, Stryker Corp. M. Hussain: 2; C; Johnson \& Johnson. G. Toth: None.

\section{E-098 MECHANICAL THROMBECTOMY IN THE EXTENDED 24 HOUR TIME WINDOW FOR ACUTE ISCHEMIC STROKE PATIENTS WITH LARGE VESSEL OCCLUSION}

${ }^{1} \mathrm{~S}$ Salam*, ${ }^{2} \mathrm{C}$ Powers, ${ }^{2} \mathrm{~S}$ Nimjee, ${ }^{2} \mathrm{P}$ Youssef, ${ }^{3} \mathrm{~S}$ Heaton, ${ }^{1} \mathrm{~V}$ Lee. ${ }^{1}$ Neurology, The Ohio State University, Columbus, $\mathrm{OH}^{2}{ }^{2}$ Neurosurgery, The Ohio State University, Columbus, $\mathrm{OH}$; ${ }^{3}$ The Ohio State University, Columbus, $\mathrm{OH}$

\subsection{6/neurintsurg-2020-SNIS.131}

Introduction In 2018 the AHA guidelines were updated to include expansion of the mechanical thrombectomy (MT) window for acute ischemic stroke (AIS) patients with anterior circulation large vessel occlusion (LVO) from 6 to 24 hours. We report our institution's clinical experience with the expanded 24 hour MT window.

Methods Among 166 AIS patients with LVO who underwent MT from January 1, 2018 to July 1, 2019 at our comprehensive stroke program, we identified 77 patients (46\%) with anterior circulation LVO treated in the extended 24 hour time window. Data was abstracted on demographics, time of Last Known Normal (LKN), National Institutes of Health Stroke Scale (NIHSS), CT brain perfusion (CTP), cerebral blood flow (CBF), time to maximum (Tmax), clinical presentation, Thrombolysis in Cerebral Infarction (TICI) score, and outcomes. Symptomatic hemorrhage was defined as neurological worsening within 36 hours accompanied by an NIHSS score increase $\geq 4$ with correlative imaging. Good functional outcome was defined as 90 day modified Rankin Score of $\leq 2$.

Results Among 77 AIS patients with anterior circulation LVO who underwent MT in the expanded window, the mean age was 66.7 years (range, 26 to 98), 39 (51\%) were female, and $69(90 \%)$ were white. Stroke risk factors included atrial fibrillation 48 (62\%), diabetes 53 (69\%), and hypertension 53 (69\%). Only $10(13 \%)$ received intravenous thrombolysis. Initial mean NIHSS was 14.5 (range 1 to 28). CTP was available in $72(94 \%)$ and the mean time from LKN to CTP was 12.0 hours (range, 2.8 - 22.8 hours). CTP mean CBF was 22.8 $\mathrm{mL} / 100 \mathrm{~g} / \mathrm{min}$ (range, 0 - $157 \mathrm{~mL} / 100 \mathrm{~g} / \mathrm{min}$ ). CTP mean Tmax was $113.6 \mathrm{~s}$ (range, 0 to 289 s). Mean time from LKN to MT groin puncture was 12.9 hours (range, 6.1 to 23.5) and $69(90 \%)$ achieved TICI 2B or 3 recanalization. Symptomatic hemorrhage occurred in 5 (6.5\%). Fifteen patients (19.5\%) expired. Among the 62 patients who survived, the mean discharge NIHSS was 8.8 (range, 0 to 26). Good functional outcome occurred in 19 (24\%).

Conclusions AIS patients with anterior circulation LVO treated in the expanded 24 hour MT window account for nearly half of our center's MT volume. Although high revascularization rates and low symptomatic hemorrhage rates were observed similar to the clinical trials, good functional outcome was lower than the trials.

Disclosures S. Salam: None. C. Powers: None. S. Nimjee: None. P. Youssef: None. S. Heaton: None. V. Lee: None.

\section{E-099 PARTICLE ANALYSIS OF A NOVEL LIQUID EMBOLIC}

I Smith*. Northern Arizona University, Flagstaff, AZ

10.1136/neurintsurg-2020-SNIS.132

Introduction Endovascular devices are becoming more widely accepted ischemic stroke treatment options in patient healthcare. Current device testing methods must be developed to quantify downstream particulate migration. In vivo models are 